\title{
Acute Endovascular Management of a Ruptured Choroidal Artery Arteriovenous Malformation in a Peripartum COVID-19-Positive Patient
}

Gaurav Tyagi1,® Karthik Kulanthaivelu $\quad$ Jitender Saini ${ }^{2} \quad$ Aravind Gowda $^{2}$ Dwarakanath Srinivas ${ }^{1}$

${ }^{1}$ Department of Neurosurgery, NIMHANS, Bengaluru, Karnataka, India

2Department of Neuroimaging and Intervention Radiology, NIMHANS, Bengaluru, Karnataka, India

Address for correspondence Gaurav Tyagi, MCh, Department of Neurosurgery, II Floor, Faculty Block, NIMHANS, Hosur Road, Bengaluru 560029, Karnataka, India (e-mail: dr_gauravtyagi@yahoo.com).

Indian J Neurosurg 2022;11:277-279

\begin{abstract}
Coronavirus disease 2019 (COVID-19) pandemic has led to significant reduction in specialty care causing delayed presentations and decreased resources for elective procedures. We present here a case of a 29 -year-old female, 34 weeks primigravida who presented with a twice ruptured right choroidal artery arteriovenous malformation (AVM). She underwent an immediate external ventricular drain placement followed by an emergency cesarean. She underwent a diagnostic angiogram showing a right choroidal AVM with a feeder artery aneurysm along with small supply to its cortical component by the M4 segment of right middle cerebral artery, draining into the basal vein of Rosenthal and vein of Labbe. The patient underwent embolization of the aneurysm and the choroidal feeders. She improved symptomatically following the procedure

Keywords

- arteriovenous malformation

- AVM in pregnancy

- choroidal AVM

- COVID-19

- endovascular management of AVM

- ruptured AVM with external cerebrospinal fluid diversion for 5 days. At follow-up magnetic resonance imaging, the residual AVM remained stable and is planned for Gamma knife radiosurgery. The newborn was taken care at a neonatal intensive care unit and was started on breastfeed on 16th day of birth once the mother had a negative COVID-19 reverse transcription polymerase chain reaction. In this study, we highlight our experience with the above-mentioned patient profile, the multidisciplinary effort during the pandemic, and the measures taken (isolation ward, COVID-19 dedicated Cath-lab and personal precautions) and advised for tackling COVID-19 patients for endovascular procedures.
\end{abstract}

\section{Introduction}

The ongoing pandemic has led to a significant reduction in other specialty care from outpatient visits to elective surgeries. ${ }^{1,2} \mathrm{~A}$ ruptured arteriovenous malformation (AVM) is a neurosurgical emergency requiring urgent diagnostic/therapeutic intervention. The risks of rupture increase with pregnancy, especially in the presence of feeder artery aneurysms., ${ }^{3,4}$ The initial goal for the management of any ruptured AVM in pregnancy is to minimize the risk of rebleeding until delivery. ${ }^{5}$

Dol https://doi.org/ $10.1055 / \mathrm{s}-0041-1735379$ ISSN 2277-954X
In this report, we present the emergent endovascular management of a feeder aneurysm in a reruptured choroidal AVM in a third trimester pregnant against a background of coronavirus disease 2019 (COVID-19).

\section{Case Report}

A 29-year-old female, 34 weeks primigravida, presented with sudden onset headache with recurrent vomit 1 week back. She lapsed into altered sensorium; her computed

\footnotetext{
(c) 2022. Neurological Surgeons' Society of India.

This is an open access article published by Thieme under the terms of the Creative Commons Attribution-NonDerivative-NonCommercial-License, permitting copying and reproduction so long as the original work is given appropriate credit. Contents may not be used for commercial purposes, or adapted, remixed, transformed or built upon. (https://creativecommons.org/licenses/by-nc-nd/4.0/).

Thieme Medical and Scientific Publishers Pvt. Ltd. A-12, 2nd Floor, Sector 2, Noida-201301 UP, India
} 
tomography (-Fig. 1A) and magnetic resonance imaging (MRI) brain showed a ruptured medial temporal AVM with intraventricular hemorrhage (IVH) and hydrocephalus. She had a history of a primary IVH with mild hydrocephalus at 4 weeks back, which was managed conservatively. This time a right frontal external ventricular drain (EVD) was placed, followed by an emergency cesarean section. The child was kept in neonatal intensive care unit and did not suffer any perinatal insult. During her peripartum course, she developed COVID-19 infection and was referred for further management at our institute.

She was admitted in the COVID-19 intensive care unit at our institute. An informed consent was taken for diagnostic and/or therapeutic angiogram. The procedure was performed in a biplane suite earmarked for COVID-19 patients with restricted operators and support staff. The AVM nidus measured $\sim 12.3 \times 2.1 \times 2.8 \mathrm{~mm}$ (-Fig. 1B and C) and the main feeders were arising from the right anterior choroidal artery with a proximal flow-related aneurysm (likely source of bleeding). Small feeders from the right M4 segment also supplied the cortical component of the AVM. Venous drainage was through the basal vein of Rosenthal into the straight sinus and the right vein of Labbe into the transverse sinus. A targeted embolization of the aneurysm and the choroidal component of the AVM was planned. A NeuronMax (Penumbra Inc, Alameda, California, United States) guiding sheath was

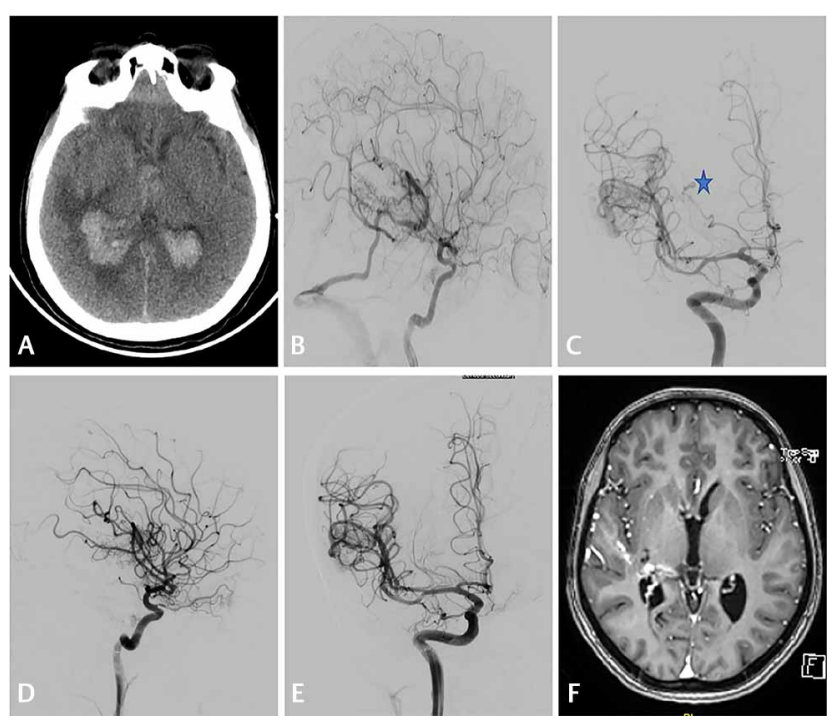

Fig. 1 (A) Axial noncontrast computed tomography brain shows intraventricular hemorrhage with largest clot volume in the right temporal horn. (B and $\mathbf{C}$ ) Diagnostic angiogram of the right internal carotid artery shows right choroidal arteriovenous malformation (AVM) with an associated cortical component with feeders from the right anterior choroidal artery with a proximal flow-related aneurysm $\left({ }^{*}\right)$ and M4 segment of the right middle cerebral artery (MCA). Venous drainage was in right basal vein of Rosenthal into the straight sinus and right vein of Labbe into the transverse sinus. (D and $\mathbf{E}$ ) Postembolization angiogram showed complete occlusion of aneurysm and choroidal component of the AVM. Residual cortical component was seen filling form the M4 branches of the MCA. (F) Follow-up magnetic resonance imaging at 3 months showed no new hemorrhage, reduction in the mass effect, and nidal dimensions. parked in the distal cervical internal carotid artery (ICA). Due to extreme tortuosity of the anterior choroidal artery, multiple attempts to cannulate it with 1.2-Fr Magic microcatheter (Balt, Montmorency, France) over 0.007" Hybrid micro wire (Balt, Montmorency, France) met with failure. Eventually, a Hyperform balloon ( $4 \times 7 \mathrm{~mm}$ ) over Expedion 0.010 microwire (Covidien/eV3, Irvine, California, United States) was inflated into the terminal ICA and M1 middle cerebral artery (MCA) to facilitate cannulation of the anterior choroidal artery. After satisfactory position of the microcatheter, under fluoroscopy guidance $0.6 \mathrm{~mL}$ of $17 \%$ glue was injected into the nidus and aneurysm. Postembolization angiogram showed complete occlusion of the aneurysm and the choroidal component of the AVM. Residual cortical component was seen filling form the M4 branches of the MCA ( - Fig. 1D and $\mathbf{E}$ ).

Her EVD was removed on postprocedure day 5 and she was discharged in a stable condition with mild asymptomatic IVH and a negative COVID-19 report. The child was managed on formula feeds under neonatologist consultation and breastfeeding was initiated on 16th day of birth. At 3 months follow-up, patient was doing well with no changes in the residue on follow-up MRI ( - Fig. 1F). She is planned for Gamma knife radiosurgery for the residual lesion later.

\section{Discussion}

Ruptured AVMs are associated with superior outcomes when managed at a high-volume center. ${ }^{6}$ The availability of a multidisciplinary expertise (neurosurgeons, intervention neuroradiologists, intensivists, and neuro-rehab care) with relatively more experience of managing AVMs even during the pandemic was an important factor for the transfer of patient to our institute. In a recent survey by the Society of Vascular and Interventional Neurology, and the World Federation of Interventional and Therapeutic Neuroradiology, it was realized that the centers offering emergent neurointerventional services across the world remained comparable to the pre-COVID era (96\%), albeit with limited resources. A substantial decrease (in up to two-third of the centers) in emergency procedures has been reported. ${ }^{7}$

The presence of a proximal aneurysm on the feeding artery ( $5 \%$ of all AVMs) is an independent risk factor for rupture. ${ }^{3}$ Such unsecured proximal aneurysms carry a higher risk of postradiosurgical bleeding. ${ }^{8}$ Similarly, the rupture rates of AVMs in pregnancy are considered to be higher than the normal population. ${ }^{4}$ These factors have to be balanced against the risks of anesthetic agents, hemodynamic changes, iodinated contrasts, and radiation exposure to the fetus while planning for the management. ${ }^{5}$ The management of the pregnancy should be neurological and not obstetric in case of a ruptured AVM with flow-related aneurysm. However, every effort should be made to save the fetus. Considering the above risk factors and the presence of viable fetus in our case, an emergency cesarean section was done, and the patient was taken up for endovascular embolization of the AVM.

The prescribed angiography suite precautions involve restricting staff numbers to essential personnel only, earmarking all interventions in a Cath lab for COVID-19 
patients only, optimizing the utilization of consumables, facilitating decontamination of frequently used equipment (e.g., angiography table controls) by wrapping with plastic sheets and disinfectants, meticulous donning and doffing of personal protective equipment in designated areas, ventilating and disinfecting the suite..$^{9}$ Adoption of telecommunication with livestreaming of the angiography video for mentoring has only helped the cause. ${ }^{7}$ In the index case, the approach to the neurointervention was tailored to acutely tackle the imminent risk of rerupture, namely the ruptured feeding artery aneurysm, and defer the management of the cortical component for a later elective sitting.

\section{Conclusion}

This case represents the multidisciplinary collaborative effort and the challenges faced by the treating team at the time of an ongoing pandemic. The patient required shifting through intensive care wards, operation theaters, and angio-suites maintaining strict isolation and protection for the healthcare workers. The care of the newborn separated from the mother, isolation, and management of the patient for COVID-19, cerebrospinal fluid diversion, and urgent endovascular embolization of the flow-related aneurysm were promptly carried out to help get over the emergency situation.

\section{Note}

A written informed consent was taken from the patient prior to the procedure that included a consent to use of anonymized data for scientific purposes.

\section{Funding}

None.

\section{Conflict of Interest} None declared.

\section{References}

1 Jean WC, Ironside NT, Sack KD, Felbaum DR, Syed HR. The impact of COVID-19 on neurosurgeons and the strategy for triaging non-emergent operations: a global neurosurgery study. Acta Neurochir (Wien 2020;162(6):1229-1240

2 Deora H, Dange P, Patel K, et al. Management of neurosurgical cases in a tertiary care referral hospital during the COVID-19 pandemic: lessons from a middle-income country. World Neurosurg 2021;148:e197-e208

3 Westphal M, Grzyska U. Clinical significance of pedicle aneurysms on feeding vessels, especially those located in infratentorial arteriovenous malformations. J Neurosurg 2000;92(6):995-1001

4 Porras JL, Yang W, Philadelphia E, et al. Hemorrhage risk of brain arteriovenous malformations during pregnancy and puerperium in a North American cohort. Stroke 2017; 48(6):1507-1513

5 Katsuragi S, Yoshimatsu J, Tanaka H, et al. Management of pregnancy complicated with intracranial arteriovenous malformation. J Obstet Gynaecol Res 2018;44(4):673-680

6 Davies JM, Lawton MT. Improved outcomes for patients with cerebrovascular malformations at high-volume centers: the impact of surgeon and hospital volume in the United States, 2000-2009. J Neurosurg 2017;127(1):69-80

7 Fiehler J, Brouwer P, Díaz C, et al. COVID-19 and neurointerventional service worldwide: a survey of the European Society of Minimally Invasive Neurological Therapy (ESMINT), the Society of NeuroInterventional Surgery (SNIS), the Sociedad Iberolatinoamericana de Neuroradiologia Diagnostica y Terapeutica (SILAN), the Society of Vascular and Interventional Neurology (SVIN), and the World Federation of Interventional and Therapeutic Neuroradiology (WFITN. J Neurointerv Surg 2020;12(8):726-730

8 Pollock BE, Flickinger JC, Lunsford LD, Bissonette DJ, Kondziolka D. Hemorrhage risk after stereotactic radiosurgery of cerebral arteriovenous malformations. Neurosurgery 1996;38(4):652-659, discussion 659-661

9 Aggour M, White P, Kulcsar Z, Fiehler J, Brouwer P. European Society of Minimally Invasive Neurological Therapy (ESMINT) recommendations for optimal interventional neurovascular management in the COVID-19 era. J Neurointerv Surg 2020;12(6):542-544 